\title{
Effect of Epoxy Resin Emulsion on the Mechanical Properties of Oil Well Cement-Based Composites
}

\author{
Miao He, ${ }^{1,2}$ Jianjian Song $\mathbb{D}^{1,2}$ Mingbiao Xu $\mathbb{D}^{1,2}$ Lei He, ${ }^{3}$ and Peng $X u^{1,2}$ \\ ${ }^{1}$ School of Petroleum Engineering, Yangtze University, Wuhan 430100, China \\ ${ }^{2} \mathrm{Hubei}$ Cooperative Innovation Center of Unconventional Oil and Gas (Yangtze University), Wuhan 430100, China \\ ${ }^{3}$ Engineering Department of Dagang Oilfield, Tianjin 300280, China \\ Correspondence should be addressed to Jianjian Song; songjian629@yangtzeu.edu.cn and Mingbiao Xu; xumingbiao@ \\ yangtzeu.edu.cn
}

Received 26 February 2020; Revised 8 August 2020; Accepted 31 August 2020; Published 19 September 2020

Academic Editor: Claudio Mazzotti

Copyright (c) 2020 Miao He et al. This is an open access article distributed under the Creative Commons Attribution License, which permits unrestricted use, distribution, and reproduction in any medium, provided the original work is properly cited.

\begin{abstract}
Oil well cement is a brittle material, which can not ensure the long-term sealing integrity of oil and gas wells when used in cementing operations directly. As a kind of polymer emulsion material, epoxy resin emulsion has a bright future for improving the properties of the cement slurry. Epoxy resin emulsion was added to oil well cement and its workability and mechanical properties were studied, the stress-strain behavior of cement samples was evaluated, and the microstructure was observed by scanning electron microscope (SEM) and X-ray diffraction (XRD). The results show the epoxy resin emulsion used in oil well cement will affect the fluidity, but the rheological property of cement slurry with different content of resin meets the construction requirements. The resin reduces the water loss of cement paste and has no adverse effect on the thickening time. The compressive strength of cement stone decreases with the increase in resin content. When the content of resin is $6 \%$, the flexural strength and impact strength of the cement sample are the largest, and $50.7 \%$ and $20.2 \%$ higher than that of the specimen without resin, respectively, after curing for 28 days. Further comparison shows that epoxy resin emulsion can improve the mechanical properties of oil well cement better than styrene-butadiene rubber latex. Meanwhile, the resin obviously improves the deformability and decreases the elastic modulus of cement stone. Compared with pure cement, resin cement slurry has no extra hydration products, but the formation of hydrated calcium silicate is inhibited. The microstructure shows that the resin forms a polymer film in the cement matrix and interweaves with cement hydration products, thus improving the flexibility of cement paste.
\end{abstract}

\section{Introduction}

Cementing operation is important for oil and gas well construction; its fundamental purpose is to achieve interlayer sealing, support and protect casing, preventing fluid channeling between different formations under the pressure difference [1-3]. Oil well cement is the main component of cementing material, but because of its brittleness after solidification, it cannot resist downhole stress damage when used directly in cementing [4]. Therefore, it is often necessary to add modified materials to improve the properties of cement paste.

Some studies have shown that the cement slurry with polymer materials is helpful to enhance the resistance ability of oil well cement to stress and to achieve long-term sealing
[5-7]. Polymer emulsion is one of the most commonly used polymers in oil well cement. Adding polymer emulsion, such as styrene-butadiene latex and ethylene vinyl acetate copolymer emulsion, to cement slurry, the mechanical properties and durability of cement slurry can be significantly improved due to the action of the polymer $[5,8]$. The modification mechanism of polymer emulsion on the properties of cement paste is mainly the formation of the polymer film and the close combination of membrane and cement hydration products [9]. After the polymer emulsion cement is solidified, the polymer bridged the interlayer crystals and bound these hydrated crystals together. In addition, the cohesive force of the modified cement is improved, forming a more cohesive microstructure and reducing the number of microcracks inside the cement matrix 
[10]. However, many polymer emulsions are prone to demulsification when subjected to mechanical force and high temperature environment, resulting in an unstable performance of cement slurry. They need to be used with stabilizers in order to be used in cement.

Epoxy resin emulsion is a kind of polymer emulsion, and it can be applied in cement-based composites without stabilizer. The epoxy resin emulsion has a wide application in the field of concrete. Ohama et al. [11] studied the strength development of the cement mortar modified by the epoxy resin emulsion. The maximum flexural strength of the polymer mortar can reach 2.1 times of the unmodified mortar, but at the same time, its compressive strength decreases and the increase of the polymer content reduces the hardening degree of the mortar. Ariffin et al. [12] studied the effect of bisphenol A epoxy resin on the performance of cement mortar without a curing agent. When the content of resin is $10 \%$, the flexural strength and splitting tensile strength of cement stone are the largest. The addition of resin makes the structure of cement stone more compact and improves the mechanical properties and durability. Mahmoud et al. [13] synthesized water-soluble sulfonated acetophenone formaldehyde resin in the laboratory, and the resin improved the compressive strength of the cement stone and reduced the porosity and water absorption of the cement stone. These investigations show that the resin improves the mechanical properties and durability of cement paste. However, the application environment of oil well cement is often accompanied by high temperature and high pressure, the epoxy emulsion used in concrete can not be directly applied to oil well cement, resulting in its use being restricted. Zhang et al. [14] have studied the effect of resin emulsion on the hydration of oil well cement, but the mechanical properties of resin emulsion cement slurry have not been systematically studied. The mechanical properties of oil well cement slurry are closely related to its construction quality.

In this research, an epoxy resin emulsion was prepared, and the effect of the epoxy resin emulsion on the mechanical properties and microstructure of the oil well cement was studied, which is helpful for the design and development of high-performance polymer cement slurry system.

\section{Experimental}

2.1. Materials. Oil well cement is a conventional class G Portland cement and obtained from Gezhouba Special Cement Co., Ltd, China. Filtration reducer, dispersant, enhancer, and retarder were procured from Jingzhou Jiahua Technology Co., Ltd, China. Epoxy resin emulsion is produced in the laboratory.

2.2. Specimen Preparation. Preparation of the cement sample was conducted regarding the Chinese standard test protocol GB 10238-2005. The specimen was prepared using a constant speed agitator (TG-3060, Shenyang Taige Oil Equipment CO., LTD, China). Filtration reducer and enhancer were utilized to reduce the water loss of cement slurry and enhance the compressive strength, respectively. Consecutively, dispersant and retarder were used to improve the fluidity and thickening time of cement slurry. Lastly, epoxy resin emulsion was used to improve the mechanical properties of the cement slurry. Table 1 lists the specific compositions of the resin modified cement slurry.

\subsection{Test Methods}

2.3.1. Workability. The workability of cement slurry was tested according to the Chinese standard test protocol GB/T 19139-2012. After curing $20 \mathrm{~min}$ in atmospheric consistometer (TG-1250, Shenyang Taige Oil Equipment CO., LTD, China) at $90^{\circ} \mathrm{C}$ and $0.1 \mathrm{MPa}$, the rheological properties were tested using a rheometer (OFITE900, OFITE, USA); the readings of different shear rates $(600 \mathrm{r} / \mathrm{min}, 300 \mathrm{r} / \mathrm{min}$, $200 \mathrm{r} / \mathrm{min}, 100 \mathrm{r} / \mathrm{min}, 6 \mathrm{r} / \mathrm{min}, 3 \mathrm{r} / \mathrm{min}$ ) could be obtained. The thickening time was measured by pressurized consistometer (TG-8040DA, Shenyang Taige Oil Equipment CO., LTD, China) at $90^{\circ} \mathrm{C}$ and $45 \mathrm{MPa}$. HPHT Filter Press (TG-71, Shenyang Taige Oil Equipment CO., LTD, China) was used to test the fluid loss of cement slurry at $90^{\circ} \mathrm{C}$ and 6.9 $\mathrm{MPa}$ for $30 \mathrm{~min}$.

2.3.2. Calculation of Rheological Parameters. The rheological properties of cement slurry are characterized by rheological parameters. After obtaining the readings of different shear rate, the fluidity index $n$ and consistency coefficient $K\left(\mathrm{~Pa} \mathrm{~s}^{n}\right)$ can be calculated according to the Chinese standard SY/T 5504.3-2008. The calculation formula is as follows:

$$
\begin{aligned}
& n=2.096 \lg \left(\frac{\Phi_{300}}{\Phi_{100}}\right), \\
& K=\frac{0.511 \Phi_{300}}{511^{n}},
\end{aligned}
$$

where $\Phi_{100}$ and $\Phi_{300}$ are the readings at a shear rate of $100 \mathrm{r} /$ $\mathrm{min}$ and $300 \mathrm{r} / \mathrm{min}$, respectively. The larger fluidity index $n$ shows the fluidity of cement slurry is better; the greater consistency coefficient $K$ indicates the cement slurry is thicker.

2.3.3. Mechanical Properties. In order to examine the mechanical properties of cement stone, all the samples were cured by a pressurized curing chamber (TG-7370D, Shenyang Taige Oil Equipment CO., Ltd., China) at $90^{\circ} \mathrm{C}$ and $21 \mathrm{MPa}$. Compressive strength and flexural strength were tested by full-automatic flexural and compression testing machine (YAW-300C, Ji'nan Zhongluchang Testing Machine Manufacturing Co., Ltd., China). The cured samples $(50.8 \mathrm{~mm} \times 50.8 \mathrm{~mm} \times 50.8 \mathrm{~mm})$ were used for examining the compressive strength at a constant loading rate of $72 \mathrm{kN} / \mathrm{min}$, the cured rectangular stones $(40 \mathrm{~mm} \times 40 \mathrm{~mm} \times 160 \mathrm{~mm})$ were used to measure flexural strength (3-point bending). The impact strength was measured by pendulum impact tester (XJJY-50, Chengdeshi Shipeng Detection Equipment Co., 
TABle 1: Mix compositions of the resin modified cement slurry.

\begin{tabular}{lccccccc}
\hline Sample number & Cement $(\mathrm{g})$ & Water $(\mathrm{g})$ & Filtrate reducer $(\mathrm{g})$ & Enhancer $(\mathrm{g})$ & Dispersant $(\mathrm{g})$ & Retarder $(\mathrm{g})$ & Epoxy resin emulsion $(\mathrm{g})$ \\
\hline R0 & 800 & 352 & 20 & 12 & 4.8 & 4.8 & 0 \\
R1 & 800 & 352 & 20 & 12 & 4.8 & 4.8 & 24 \\
R2 & 800 & 352 & 20 & 12 & 4.8 & 4.8 & 48 \\
R3 & 800 & 352 & 20 & 12 & 4.8 & 4.8 & 72 \\
\hline
\end{tabular}

Ltd., China) and the size of samples was $10 \mathrm{~mm} \times 15 \mathrm{~mm} \times 120 \mathrm{~mm}$.

2.3.4. Stress-Strain Behavior. After curing for $28 \mathrm{~d}$, the uniaxial stress-strain behavior of the sample was determined by a universal testing machine (HY-20080, Shanghai Hengyi Precision Instrument Co., Ltd., China) according to the Chinese standard test protocol GB/T 50266-2013. The cement stone was compressed at a constant loading rate of $2 \mathrm{kN} / \mathrm{min}$.

2.3.5. Phase Analysis. X-ray diffraction (XRD) analysis was carried out using a Bruker D8-Advance X-ray diffractometer. The measurement data was obtained in the $2 \theta$ range from $5^{\circ}$ to $90^{\circ}$ (the accumulation grade is $0.02^{\circ}$ ) and using $\mathrm{Cu}$ $\mathrm{K} \alpha$ radiation.

2.3.6. Micromorphology of Fracture Surface. The microstructure of cement stone was observed using a scanning electron microscope (SEM) (SU 8010, HITACHI, Japan). The specimen was cured for $28 \mathrm{~d}$.

\section{Experimental Results and Discussion}

3.1. Workability of Resin Emulsion Modified Cement Slurry. The workability of cement slurry mainly include rheology, thickening time, and water loss. Rheology plays a key role in determining various parameters of cementing [15]. Good rheology can ensure good pumping performance and improve the displacement efficiency of the cement slurry. The most important factor affecting the safety of construction is thickening time. Considering the safety of cementing operation, the thickening time must be measured in the same temperature and pressure of the downhole and taken as the basis of construction operation [16]. The water loss of cement slurry is the free water that can be filtered through a definite area of pores under specified temperature and pressure difference. The excessive water loss will cause premature dehydration, change the thickening time, and reduce the strength of cement paste, resulting in problems such as annulus bridge blockage and interlaminar flow [17]. The rheology, thickening time, and water loss of cement slurry without resin and with different content of resin were evaluated, and the fluidity index $n$ and consistency coefficient $\mathrm{K}$ were calculated according to formulas (1) and (2). Evaluation data of rheology is listed in Table 2. The thickening time and water loss of resin cement slurry are shown in Figures 1 and 2, respectively.

From the data in Table 2, the addition of resin will change the rheological properties of cement paste. With the increase in the amount of resin, the fluidity index of resin cement paste decreases and the consistency coefficient increases, which indicates that the resin will make the cement paste thicker. When the content of the resin is $9 \%$, fluidity index $n$ and consistency coefficient $K$ of resin cement slurry are 0.75 and 0.57 , respectively. Depending on the Chinese standard SY/T 5504.3-2008, the rheology of resin cement slurry can meet the construction requirements. As can be seen from Figure 1, the resin prolongs thickening time, but there is no adverse effect on cementing construction. It can be seen from Figure 2 that resin reduces the amount of water loss when the content is $3 \%$, but with the increase in resin content, the water loss increased. This may be that less polymer resin has a similar function with polymer filtrate reducer, which makes the filter cake structure more compact and reduces the water loss. And more resin content increases the content of the liquid phase in the cement slurry, resulting in more water loss. The experimental results demonstrate that resin will make the cement paste thicker, but it can meet the construction requirements within $9 \%$ content of resin. Moreover, a small amount of resin emulsion will reduce the water loss while excess resin content has the opposite effect, and resin emulsion has no adverse effect on thickening time.

\subsection{Mechanical Properties of Resin Modified Cement Slurry}

3.2.1. Compressive Strength of Resin Modified Cement Slurry. Compressive strength is the maximum stress to destroy the cement stone, which is the ability to maintain the integrity of cement stone under compression stress [18]. The effect of resin on compressive strength is related to the life and recovery of oil and gas wells. The compressive strength of cement stone with 0\% (R1), 3\% (R1), 6\% (R2), 9\% (R3) resin emulsion cured for different ages is illustrated in Figure 3.

As can be seen from the data in Figure 3, the compressive strength of cement paste is reduced by resin emulsion. At all curing time, the compressive strength of pure cement sample $\mathrm{R} 0$ is the largest and that of resin cement slurries is smaller. The compressive strength of resin cement samples R1, R2, and R3 cured for $1 \mathrm{~d}$ is $5.5 \%, 14.7 \%$, and $36 \%$ lower than that of $\mathrm{R} 0$, respectively. With the prolongation of curing age, the compressive strength of samples increases rapidly. After curing for 28 days, the compressive strength of resin cement sample R1, R2, and R3 is $7.6 \%, 15.5 \%$, and $24.4 \%$ lower than 
TABLE 2: Rheology of resin emulsion cement slurry.

\begin{tabular}{lcccccccc}
\hline Resin emulsion content (\%) & $\Phi_{600}$ & $\Phi_{300}$ & $\Phi_{200}$ & $\Phi_{100}$ & $\Phi_{6}$ & $\Phi_{3}$ & $n$ & \multicolumn{1}{c}{$K\left(\mathrm{~Pa} \mathrm{~s}^{n}\right)$} \\
\hline 0 & 245 & 148 & 108 & 59 & 4 & 3 & 0.84 \\
3 & $300+$ & 206 & 152 & 87 & 9 & 6 & 0.78 \\
6 & $300+$ & 217 & 157 & 94 & 11 & 9 & 0.76 \\
9 & $300+$ & 251 & 178 & 110 & 13 & 10 & 0.43 \\
\hline
\end{tabular}

Note: $\Phi_{600}, \Phi_{300}, \Phi_{200}, \Phi_{100}, \Phi_{6}$, and $\Phi_{3}$ are the shear rates of $600 \mathrm{r} / \mathrm{min}, 300 \mathrm{r} / \mathrm{min}, 200 \mathrm{r} / \mathrm{min}, 100 \mathrm{r} / \mathrm{min}, 6 \mathrm{r} / \mathrm{min}, 3 \mathrm{r} / \mathrm{min}$ respectively.

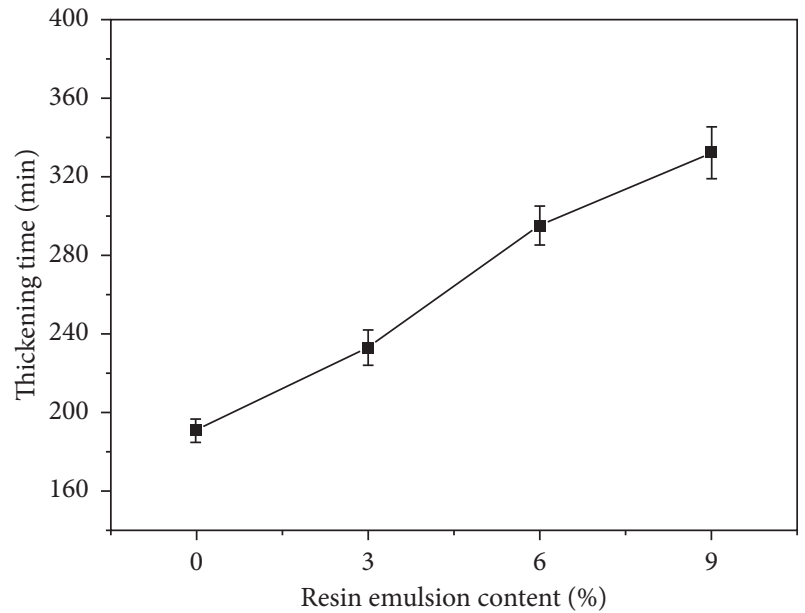

Figure 1: Thickening time of resin emulsion cement slurry.

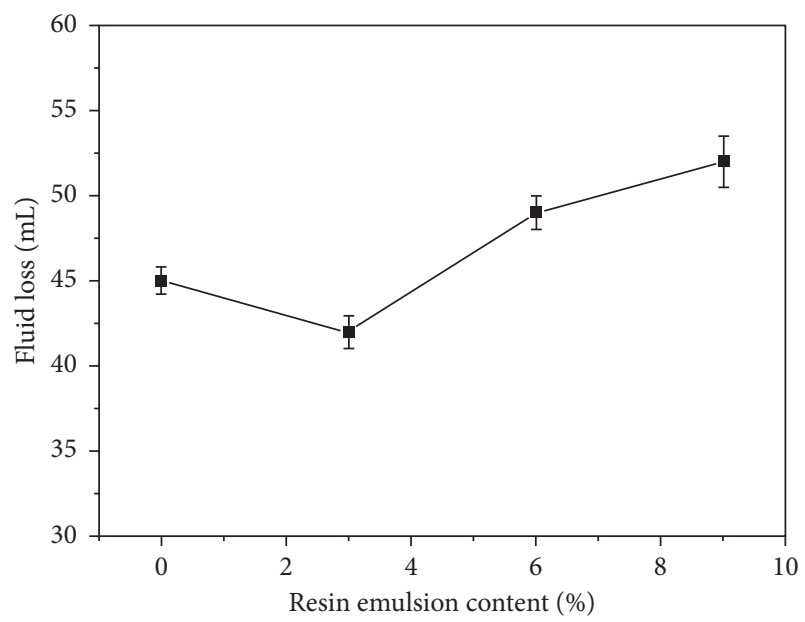

FIGURE 2: Water loss of resin emulsion cement slurry.

that of cement sample $\mathrm{R} 0$ without resin, respectively. The results show that the addition of resin emulsion reduces the compressive strength of cement samples, which is consistent with the effect of other polymer emulsion material on compressive strength.

3.2.2. Flexural Strength of Resin Modified Cement Slurry. The ductility of the cement sample can be indirectly characterized by the flexural strength, which can be obtained by a three-point bending test [19]. The flexural strength of resin cement stone was tested in the laboratory and the experimental results are shown in Figure 4.
From Figure 4, the flexural strength of cement samples increases with the increase of resin content when the content is below $6 \%$. However, when the content is more than $6 \%$, the flexural strength changes disadvantageously, and the flexural strength tends to decrease. The addition of resin emulsion can enhance the flexural strength of cement paste. After curing for 28 days, the flexural strength of R1, R2, and R3 of cement stone samples was $10.5 \%, 18.9 \%$, and $12.6 \%$, respectively, higher than that of the $\mathrm{R} 0$ sample. The flexural strength increases with the curing time. When the content of resin is $6 \%(\mathrm{R} 2)$, the flexural strength of specimens cured for $3 \mathrm{~d}, 7 \mathrm{~d}, 14 \mathrm{~d}$, and $28 \mathrm{~d}$ is $18.7 \%, 28 \%, 42.7 \%$, and $50.7 \%$ higher than that cured for $1 \mathrm{~d}$. The resin emulsion can effectively enhance the flexural strength of cement stone and improve the ability to resist the external force.

3.2.3. Impact Strength of Resin Modified Cement Slurry. Impact strength can directly reflect the toughness and quantitatively characterize the toughening effect of cement stone [20]. The impact strength is the energy consumed when cement specimen is subjected to impact force. The impact strength of resin cement paste is illustrated in Figure 5 .

It can be seen from Figure 5 that the impact strength of resin cement samples are larger than that of pure cement without resin at different curing period, and the impact strength of sample R2 is best. After curing $1 \mathrm{~d}$ and $28 \mathrm{~d}$, the impact strength of sample $\mathrm{R} 2$ is $1.8 \mathrm{~kJ} / \mathrm{m}^{2}$ and $2.14 \mathrm{~kJ} / \mathrm{m}^{2}$, respectively. When the curing time is $28 \mathrm{~d}$, the impact strength of resin cement samples R1, R2, and R3 is $16.9 \%, 20.2 \%$, and $14.6 \%$ higher than that of pure cement samples $\mathrm{R} 0$, respectively. The results show that a small amount of resin is beneficial to improve the impact strength, but an excessive amount will reduce the reinforcement effect, which is consistent with the effect of resin on flexural strength. The reason may be that a small amount of resin mixed with cement slurry has little effect on cement hydration, which can form polymer flexible structure in the cement paste and improve the performance of cement stone. However, excessive resin increases the content of nonhydrated phase in cement paste, and its contribution to the strength of cement paste decreases.

3.2.4. Comparison of the Effect of Epoxy Resin Emulsion and Styrene-Butadiene Rubber Latex. In order to study the effect of epoxy resin emulsion, $6 \%$ resin emulsion and $6 \%$ styrenebutadiene latex were added to the same cement slurry 

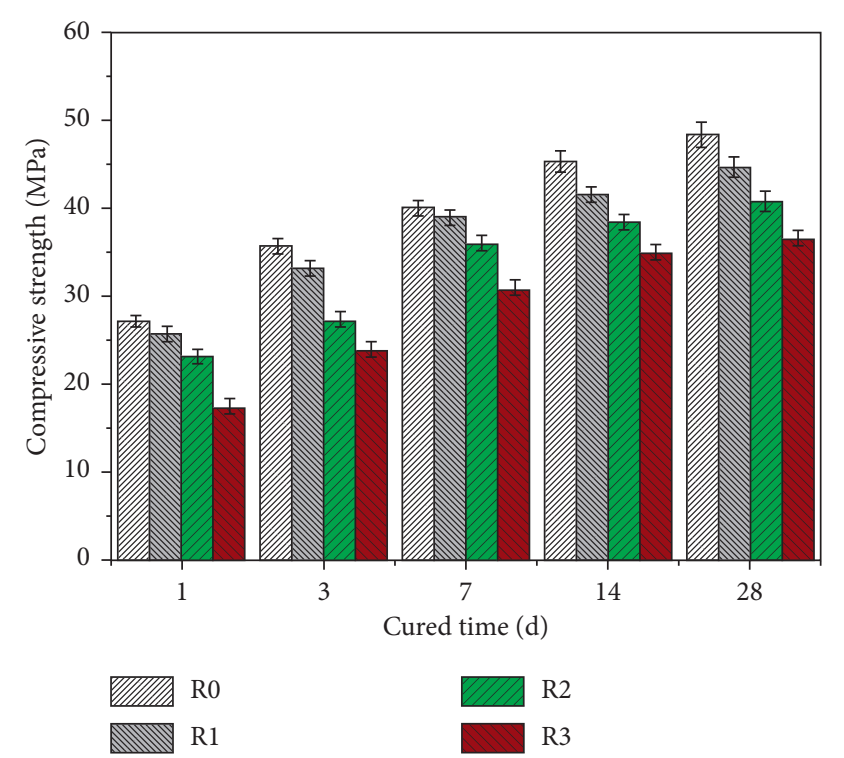

Figure 3: Compressive strength of resin modified cement slurry.

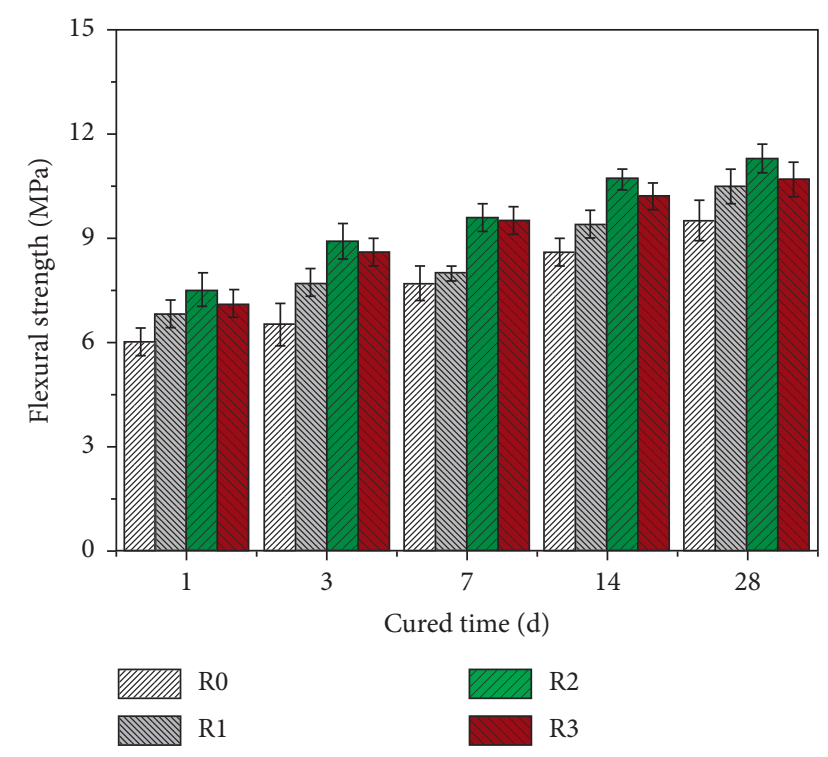

FIgURE 4: Flexural strength of resin modified cement slurry.

formula, respectively. After curing for 7 days, the mechanical properties of different cement pastes were evaluated. The experimental results are shown in Table 3.

According to the results in Table 3, the compressive strength of resin emulsion cement slurry is not significantly different from that of styrene-butadiene rubber latex cement slurry in the same curing time, and the flexural strength and impact strength of the epoxy resin emulsion cement slurry were increased by $11.6 \%$ and $7.1 \%$, respectively, compared with those of styrene-butadiene rubber latex cement slurry. Although both epoxy resin emulsion and styrene-butadiene rubber latex are flexible polymer materials, resin emulsion can improve the mechanical properties of oil well cement better.
3.3. Stress-Strain Behavior of Resin Modified Cement Sample. The previous results show that flexible resin can enhance the mechanical properties of oil well cement composite. In order to achieve long-term cementing isolation, in addition to excellent mechanical properties, the cement stone also needs to have great flexibility and deformability. The stress-strain behavior is the deformation rule of cement stone under the action of external stress. The uniaxial compression stressstrain results of resin cement and pure cement samples are shown in Figure 6.

It can be seen from Figure 6 that the differences in stressstrain behavior of resin cement and pure cement sample are large under external loading. If there is more resin emulsion added in cement paste, the deformation of cement stone is greater under the same load. From the stress-strain curve, when the cement stone is compressed, the strain increases with the increase of stress. In the constant stress range, the increase in the strain of resin cement paste is more obvious than that of pure cement paste, and the strain of R3 is larger than that of specimen R2 and R1. After reaching the maximum stress, the stress of the pure cement sample decreases rapidly, while the stress of specimen R3 decreases slowly. The reason is that at the beginning of compressing, it has a nonlinear relationship between stress and strain, with the load increasing, and then the cement stone is unstable. When running up to the maximum stress, cement stone is destroyed. However, the destruction of cement stone is a gradual process, the cement sample with good flexibility has a certain load-bearing capacity after reaching the peak stress, so the stress drops slowly. According to the test results of stress-strain behavior, the elastic modulus of cement stone can be obtained. It can be seen from the results that the maximum strain of $\mathrm{R} 3$ is increased by $71.7 \%$ and the modulus of elasticity is decreased by $54.3 \%$ compared with the pure cement R0. The experimental results show that the resin improves the deformability of cement paste and reduces the elastic modulus, which is of great significance to enhance the resistance loading capacity of cement stone in downhole and ensure the long-term cementing isolation.

3.4. Phase Analysis of the Resin Modified Cement Slurry. If the composition, group and structure of crystalline materials are different, their diffraction patterns show differences in the number of diffraction peaks, angle position, relative intensity order, and the shape of diffraction peaks. Therefore, the phase composition and structure of the samples can be identified by comparing the X-ray diffraction (XRD) patterns of the samples with those of the known crystalline materials. Crystal materials are symmetrical crystallographic systems. High crystalline materials have strong patterns and few lines, while lower crystalline systems have weaker patterns and more lines [21].

The XRD patterns of pure cement paste (R0) and resin modified cement paste (R3) are shown in Figure 7. In general, the main hydration products identified from the hydration of Portland cement are microcrystalline calcium silicate hydrates (CSH) (ICDD PDF No. 00-029-0329), ettringite (ICDD PDF No. 00-031-0251), and portlandite 

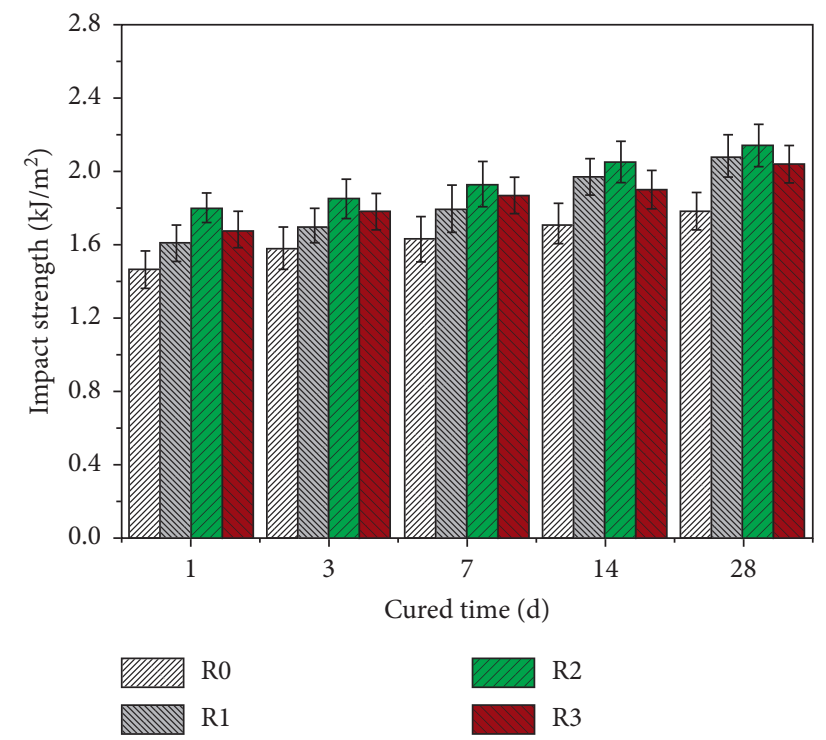

FiguRE 5: Impact strength of resin modified cement slurry.

TABLe 3: Mechanical properties of different cement pastes at 7-day cure.

\begin{tabular}{lcc}
\hline Performance & 6\% epoxy resin emulsion & 6\% styrene-butadiene rubber latex \\
\hline Compressive strength $(\mathrm{MPa})$ & 35.9 & 36.2 \\
Flexural strength $(\mathrm{MPa})$ & 9.6 & 8.6 \\
Impact strength $\left(\mathrm{kJ} / \mathrm{m}^{2}\right)$ & 1.95 & 1.82 \\
\hline
\end{tabular}

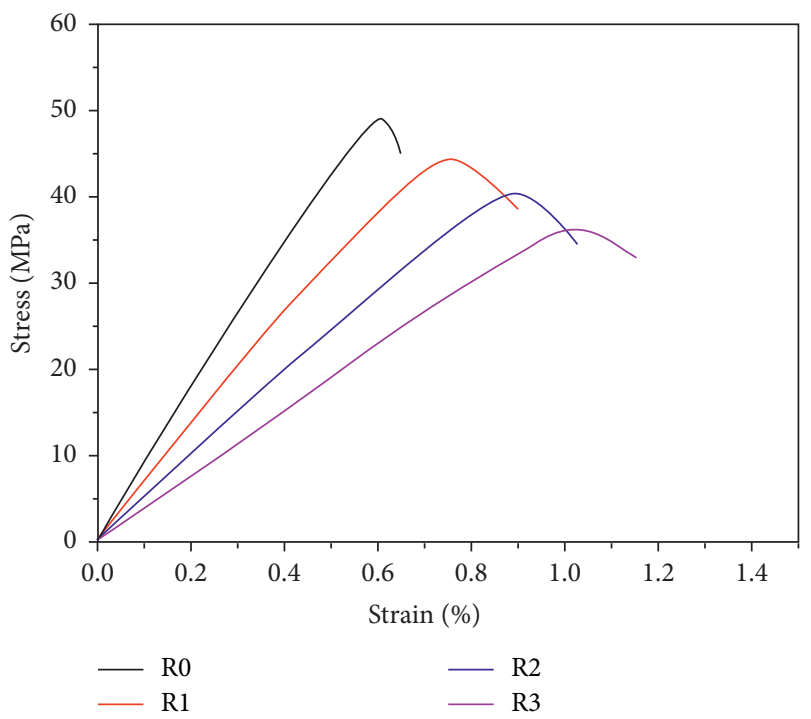

FIgURE 6: Stress-strain behavior of cement slurry.

(ICDD PDF No. 01-084-1270) [22]. For the two samples, the main characteristic peaks of XRD spectra are almost identical, indicating that the addition of resin does not cause the formation of new hydration products. The characteristic peaks of pure cement paste are obviously stronger than those of resin cement paste, which may be that the addition of resin affects the hydration process of cement paste and reduces the crystallinity of hydration products, especially
$\mathrm{CSH}\left(2 \theta=25^{\circ}-35^{\circ}\right)$ (which provides strength for the cement paste). The decrease of the crystallinity of CSH in cured resin cement slurry may be the cause of the decrease of compressive strength of resin cement paste.

3.5. Micromorphology of Fracture Surface. Figure 8 is the fracture morphology of cement paste without resin. From Figures 8(a) and 8(b), the typical hydration products, sticklike AFt, laminated $\mathrm{Ca}(\mathrm{OH})_{2}$ crystals, and amorphous $\mathrm{CSH}$ gel can be observed. Fibrous CSH gel forms a network structure framework in the cement paste. There is no polymer film in the cement matrix.

Figure 9 is the microstructure of resin cement specimen R3 observed by scanning electron microscope (SEM). As can be seen from Figure 9(a), the typical hydration products, such as amorphous CSH gel, can be observed. The three-dimensional network structure with $\mathrm{CSH}$ gel plays a role in supporting the skeleton. The polymer film is dispersed in the hydration products. This membrane is the main form of resin in the cement stone. Figure 9 shows that the hardened cement slurry formed by the netting structure is interwoven with numerous CSH gel, which is embedded with the crystalline phase of hydration products. The formation of CSH gel is the process of hydration products from dissolution to coalescence $[23,24]$, the polymer film can be cemented with $\mathrm{CSH}$ gel to form an interpenetrating structure when the slurry is solidified. At the macroscale, the brittleness of the cement sample is decreased and the 


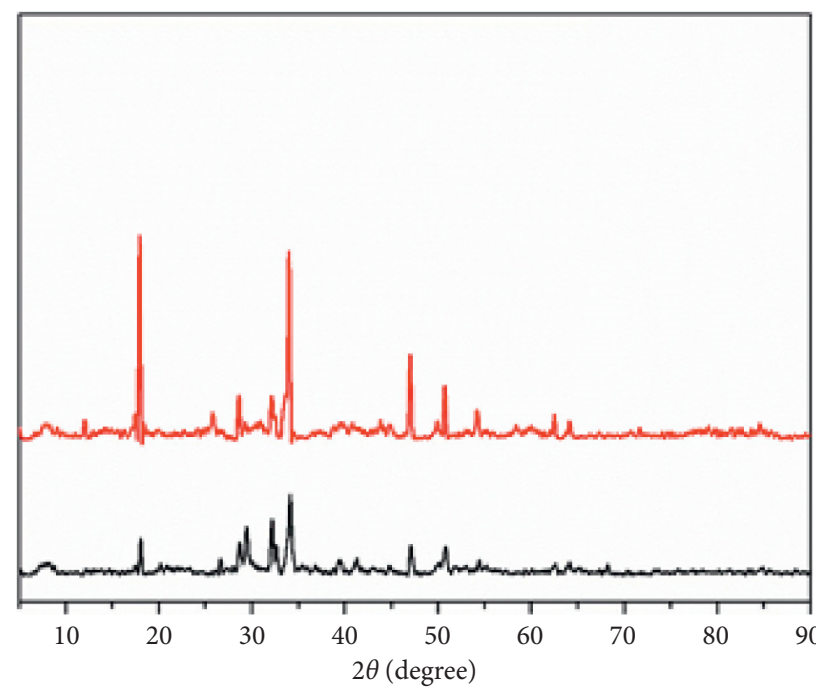

— R0

FIGURE 7: XRD patterns of pure cement paste (R0) and resin modified cement paste (R3).

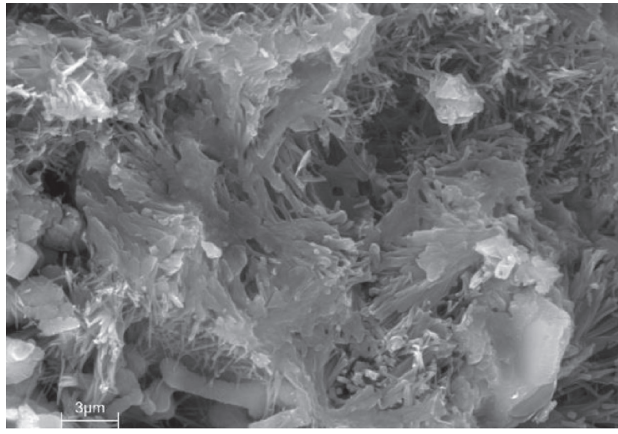

(a)

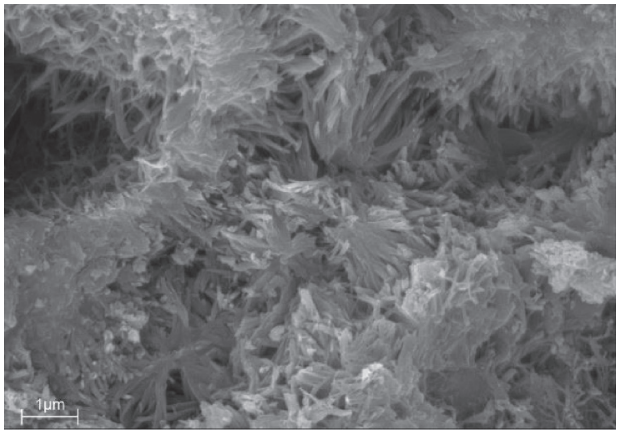

(b)

Figure 8: Micromorphology of cement sample R0.

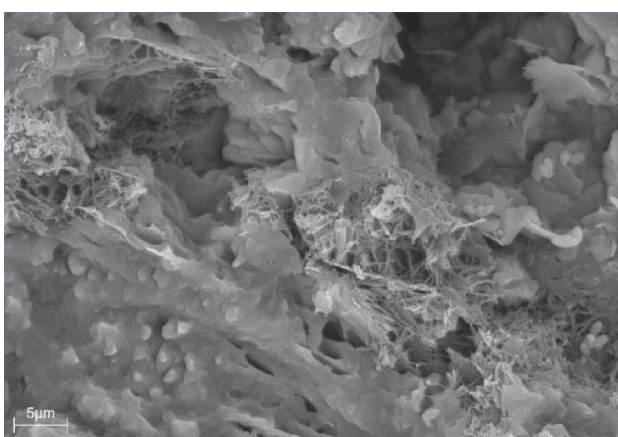

(a)

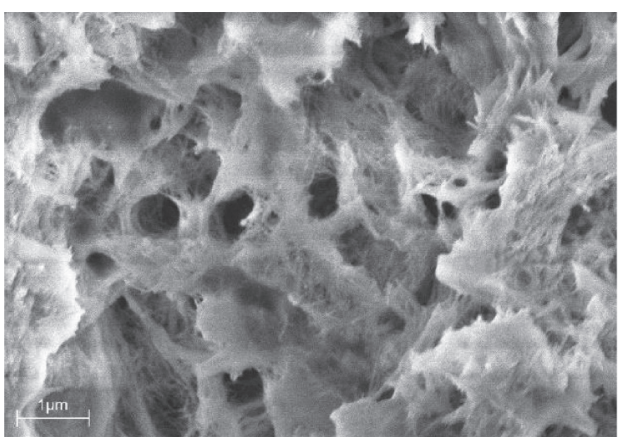

(b)

Figure 9: Micromorphology of resin modified cement sample R3. 
elasticity increases because of the embedding of a flexible polymer membrane.

\section{Conclusion}

Based on the experimental results, the following conclusions can be obtained:

(1) Epoxy resin emulsion increases the rheological value of the cement paste, but the rheological properties meet the construction requirements. In addition, the resin increases water loss and has an irregular effect on the thickening time.

(2) Resin emulsion reduced the compressive strength of cement paste, but greatly increased its flexural strength and impact strength. In addition, too much resin (above 6\%) has a negative impact on the mechanical properties of cement paste. Epoxy resin emulsion can improve the mechanical properties of oil well cement better than styrene-butadiene rubber latex.

(3) Compared with the pure cement sample, the strain of the resin cement slurry is increased, the elastic modulus is significantly decreased, and it has better deformation ability.

(4) No extra hydration products were produced after the resin was added to the cement slurry, but the formation of CSH was inhibited, which is the reason for the decrease in the compressive strength of the resin cement paste. The interlaced combination of resin polymer membrane and cement hydration leads to the decrease of the elastic modulus and the increase of flexibility of the cement slurry.

\section{Data Availability}

The data used to support the findings of this study are included within the article.

\section{Conflicts of Interest}

The authors declare that they have no conflicts of interest.

\section{Acknowledgments}

Financial support was provided for this research by National Natural Science Foundation of China (Nos. 51804044 and 51904034) and National Science and Technology Major Project (No. 2017ZX05032004-004).

\section{References}

[1] L. Boukhelifa, N. Moroni, S. G. James, S. Le Roy-Delage, M. J. Thiercelin, and G. Lemaire, "Evaluation of cement systems for oil and gas well zonal isolation in a full-scale annular geometry," SPE Drilling \& Completion, vol. 20, pp. 44-53, 2004.

[2] M. Choolaei, A. M. Rashidi, M. Ardjmand, A. Yadegari, and H. Soltanian, "The effect of nanosilica on the physical properties of oil well cement," Materials Science and Engineering: A, vol. 538, pp. 288-294, 2012.

[3] D. Bakirov, V. Burdyga, M. Fattakhov, and A. Belousov, "Developing cement mixtures to cement horizontal well intervals with multi-stage fracturing," in Proceedings of the SPE Russian Petroleum Technology Conference, Moscow, Russia, October 2019.

[4] J. Song, M. Xu, W. Liu et al., "Thermoplastic rubber (TPR) modified by a silane coupling agent and its influence on the mechanical properties of oil well cement pastes," Advances in Materials Science and Engineering, vol. 2019, Article ID 3587081, 11 pages, 2019.

[5] R. Morlat, G. Orange, Y. Bomal, and P. Godard, "Reinforcement of hydrated portland cement with high molecular mass water-soluble polymers," Journal of Materials Science, vol. 42, no. 13, pp. 4858-4869, 2007.

[6] M. D. Hudson, P. Sheperd, and J. Ricci, "Flexible cement slurry survives multistage hydraulic fracturing treatment," in Proceedings of the SPE Unconventional Resources Conference, February 2017.

[7] R. H. Williams, D. K. Khatri, R. F. Keese et al., "Flexible, expanding cement system (FECS) successfully provides zonal isolation across marcellus shale gas trends," in Proceedings of the Canadian Unconventional Resources Conference, November 2011.

[8] Z. Lu, X. Kong, Q. Zhang et al., "Influences of styrene-acrylate latexes on cement hydration in oil well cement system at different temperatures," Colloids and Surfaces A: Physicochemical and Engineering Aspects, vol. 507, pp. 46-57, 2016.

[9] J. E. Isenburg and J. W. Vanderhoff, "Hypothesis for reinforcement of portland cement by polymer latexes," Journal of the American Ceramic Society, vol. 57, no. 6, pp. 242-245, 1974.

[10] E. Knapen and D. Van Gemert, "Cement hydration and microstructure formation in the presence of water-soluble polymers," Cement and Concrete Research, vol. 39, no. 1, pp. 6-13, 2009.

[11] Y. Ohama, M. Ochi, S. Kumagai et al., "Strength developmentand epoxy resinement interaction in hardenedree epoxy modified mortars," in Proceedings of International Symposium on Brittle Matrix Composites 8, A. M. Brandt, V. C. Li, andL. H. Marshall, Eds.,pp. 315-322, ZTUREK RSI and Woodhead Publication, Warsaw, Poland, 2006.

[12] N. F. Ariffin, M. W. Hussin, A. R. M. Sam et al., "Strength properties and molecular composition of epoxy-modified mortars," .Construction and Building Materials, vol. 94, pp. 315-322, 2015.

[13] A. A. M. Mahmoud, M. S. H. Shehab, and A. S. El-Dieb, "Concrete mixtures incorporating synthesized sulfonated acetophenone-formaldehyde resin as superplasticizer," $\mathrm{Ce}$ ment and Concrete Composites, vol. 32, no. 5, pp. 392-397, 2010.

[14] Z. Zhang and P. Yan, "Hydration kinetics of the epoxy resinmodified cement at different temperatures," Construction and Building Materials, vol. 150, pp. 287-294, 2017.

[15] A. Shahriar and M. L. Nehdi, "Effect of supplementary cementitious materials on rheology of oil well cement slurries," Advances in Civil Engineering Materials, vol. 3, no. 1, pp. 454-478, 2014.

[16] G. Bassioni and M. M. Ali, "The effect of counterion in lignosulfonates as oil-well cement retarders," Advances in Cement Research, vol. 25, no. 5, pp. 245-253, 2013. 
[17] K. Jiang, "Effect of powdered acrylonitrile-butadiene rubber (PNBR)on the properties of cement slurry," Oilfield Chemistry, vol. 36, no. 4, pp. 604-609, 2019.

[18] O. A. Falode, K. K. Salam, A. O. Arinkoola, and B. M. Ajagbe, "Prediction of compressive strength of oil field class G cement slurry using factorial design," Journal of Petroleum Exploration and Production Technology, vol. 3, no. 4, pp. 297-302, 2013.

[19] S. T. Kang, B. Yeon Lee, J.-K. Kim, and Y. Y. Kim, "The effect of fibre distribution characteristics on the flexural strength of steel fibre-reinforced ultra high strength concrete," Construction \& Building Materials, vol. 25, no. 5, pp. 2450-2457, 2011.

[20] G. Ramakrishna and T. Sundararajan, "Impact strength of a few natural fibre reinforced cement mortar slabs: a comparative study," Cement and Concrete Composites, vol. 27, no. 5, pp. 547-553, 2005.

[21] M. I. Neves, V. Oliva, B. Mrabet et al., "Surface chemistry of cement pastes: a study by x-ray photoelectron spectroscopy," Surface \& Interface Analysis, vol. 33, no. 10-11, pp. 834-841, 2002.

[22] A. R. Cestari, E. F. S. Vieira, and F. C. da Rocha, "Kinetics of interaction of hardened oil-well cement slurries with acidic solutions from isothermal heat-conduction calorimetry," Thermochimica Acta, vol. 430, no. 1-2, pp. 211-215, 2005.

[23] L. Liu, Y. Zhang, W. Zhang, L. Zhiyong, and Z. Lihua, "Investigating the influence of basalt as mineral admixture on hydration and microstructure formation mechanism of cement," Construction \& Building Materials, vol. 48, no. 19, pp. 434-440, 2013.

[24] J. Han, K. Wang, J. Shi, and Y. Wang, "Mechanism of triethanolamine on Portland cement hydration process and microstructure characteristics," Construction and Building Materials, vol. 93, pp. 457-462, 2015. 